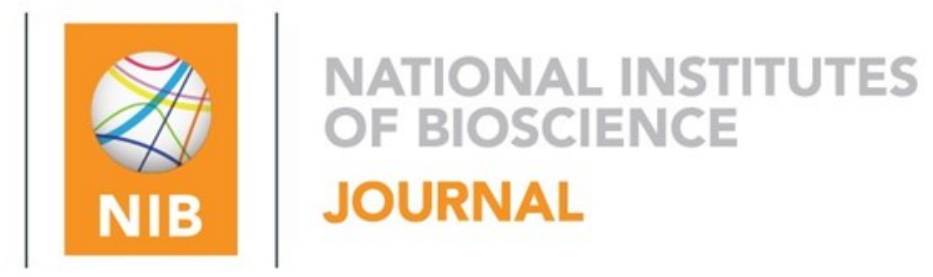

\title{
Genome Editing and the Future of Farming
}

Conference held September 6th, 2016 at The Roslin Institute, Edinburgh

\section{Gene editing: Breeding or GMO?}

\author{
Alison Van Eenennaam, PhD \\ Animal Genomics and Biotechnology Cooperative Extension Specialist \\ Department of Animal Science \\ 2113 Meyer Hall \\ One Shields Avenue \\ University of California - Davis \\ Davis, CA 95616 \\ U.S.A. \\ Corresponding author: \\ Email: alvaneenennaam@ucdavis.edu

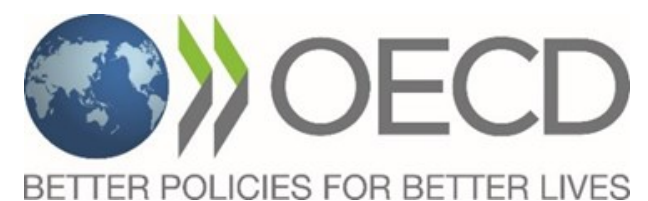

The opinions expressed and arguments employed in this publication are the sole responsibility of the authors and do not necessarily reflect those of the OECD or of the governments of its Member countries.

The Conference was sponsored by the OECD Co-operative Research Programme on Biological Resource Management for Sustainable Agricultural Systems, whose financial support made it possible for some of the invited speakers to participate in the Conference. 


\title{
Gene editing: Breeding or GMO?
}

\author{
Alison Van Eenennaam, PhD \\ Animal Genomics and Biotechnology Cooperative Extension Specialist \\ Department of Animal Science \\ 2113 Meyer Hall \\ One Shields Avenue \\ University of California - Davis \\ Davis, CA 95616 \\ U.S.A. \\ Corresponding author: \\ Email: alvaneenennaam@ucdavis.edu
}

\begin{abstract}
Global regulatory frameworks will soon be challenged by recent scientific developments in methods for generating genetically modified animals, particularly gene editing techniques. It is unclear whether animals produced using such technologies will fall under or outside of the regulations developed for genetically engineered $(\mathrm{GE})$ animals produced using recombinant DNA (rDNA) techniques. Many gene editing applications will result in animals that carry induced mutations in target genes or desirable alleles or sequences that originated in other breeds or individuals from within that species. As such, there will be no rDNA or transgenic construct in the animal, and no novel combination of genetic material that has been altered in a way that could not be achieved by natural mating or techniques used in traditional breeding and selection. The current regulatory approach to GE animals has had a stifling effect on the use of this technology in animal breeding programmes, and to date no GE animal has yet been sold for food purposes anywhere in the world. Given the importance of improved genetics to the overall environmental footprint of food production, precluding breeder access to safe innovations for use in genetic improvement programmes has a large opportunity cost. If genome editing is going to have a global impact on animal breeding programmes, its oversight should, ideally, be proportional to risk based on the novelty of the trait, consider and evaluate both benefits and risks, and be fit for
\end{abstract}


purpose, meaning that the reduction in risk obtained by regulatory oversight is greater than the costs of compliance.

Regulatory frameworks for genetically modified animals are concurrently being formulated in many countries in concert with rapidly advancing technologies for creating such animals, including gene editing and approaches to generate targeted gene knockouts in livestock species. These new animal breeding techniques result in genetically modified organisms (GMOs) that do not fit the classic definition of "transgenic" or genetically engineered (GE), although they are produced through human intervention using recombinant DNA (rDNA) techniques. Some groups have argued that because these genetic modifications are, for at least part of the procedure, produced outside the organism by people using in vitro techniques this alone should be the trigger for regulation (1). However, this seems to disregard the plethora of in vitro techniques that are commonly utilised in conventional animal breeding programmes (2). If risk is the main rationale for regulating genetic modification methods, there does not appear to be a clear rationale for regulating only traits and DNA sequences produced using rDNA techniques. If human intervention using in vitro techniques in breeding programmes is the trigger for regulation, this would seem to apply equally to many of the breeding methods used in the production of modern broiler chickens and high-producing milk cows which are clearly genetically modified animals relative to their wild ancestors: the jungle fowl and auroch, respectively.

\section{HOW MIGHT GENE EDITING INTERSECT WITH CONVENTIONAL BREEDING?}

Some of the large-scale genomic and sequencing projects have revealed a number of single nucleotide polymorphisms (SNPS) and haplotypes in which one naturally-occurring allele results in superior performance to that observed associated with an alternative allele. Consequently, an animal's genome could theoretically be edited to the superior allele at one or more genomic locations. To date, targeting different genes simultaneously has allowed bi-allelic modification of 
as many as three genes at once $(3,4)$. Multiple favorable alleles are rarely found in a single individual, and gene editing offers an advantage over conventional selection by efficiently increasing the frequency of desirable alleles in an individual, or even an entire breed, by moving naturally-occurring alleles without also incurring undesirable linkage drag.

In order for gene editing to be an important factor for genetic change, it must integrate smoothly into conventional animal breeding programmes and reliably edit the germline of breeding stock. Gene editing of somatic cells, followed by somatic cell nuclear transfer (SCNT) cloning, can be used to introduce changes. Alternatively, gene editing reagents can be injected directly into the cytoplasm of single cell zygotes of the next generation.

In livestock to date, the primary method to deliver nuclease-mediated genetic changes has been cell culture followed by SCNT (5). This method is advantageous because it allows for genotyping and/or screening of the gene edited cell line before it is transferred into the enucleated oocyte. This ensures that only the desired edits are made. On the downside, SCNT is associated with welldocumented drawbacks such as early embryonic losses, postnatal death and birth defects (6).

Alternatively, direct editing of single-cell zygotes offers an approach to introduce edits into the next generation, however the disadvantage is that not all of the embryos will be edited. Despite this, direct editing is more desirable than SCNT since fewer embryos are required, on average, to achieve the desired result (5). Direct editing of zygotes has successfully been used to knock-in entire interspecies allele substitutions $(7,8)$. Issues with mosaicism have been associated with this method (9-11), but approaches are being developed to minimise this problem.

Gene editing could theoretically be applied to many different traits in livestock, including known fertility impairing haplotypes (12), polled (hornless) (13) and to correct known Mendelian genetic defects (14), in conjunction with conventional selection methods to continue making progress towards a defined selection objective. It also provides a means by which the discovery of causative SNPs (Quantitative Trait Nucleotides; QTNs) through sequencing projects and the 
information obtained from various genome wide association studies (GWAS) could be translated into valuable genetic variation for use in animal breeding programmes (15). In one simulation study, response to selection was predicted to be improved four-fold after 20 generations as a result of the combined use of gene editing and traditional genomic selection (16). Gene editing will likely be used to complement conventional breeding programmes, rather than replace them.

Although these methods offer many advantages, it is important to understand that hundreds, if not thousands, of different genes and their interactions impact complex traits. As a result, not all of the genes that influence these traits have been identified, so the sequences of the desirable alleles are not always known. For now, it is likely that relatively large effect loci and known targets will be the focus of editing in efforts to correct genetic defects or decrease disease susceptibility. The backbone of breeding programmes will continue to be conventional selection in which selection for many small effect loci that impact complex traits will contribute to the breeding objective.

\section{WILL GENOME EDITING BE REGULATED?}

Now is an opportune time to review the regulatory framework for GE animals and examine whether the current approaches strike the appropriate balance between the unique risks and benefits associated with this technology. Regulatory oversight does not apply to animal breeding per se, although the sale of an unsafe food product is illegal regardless of the breeding methods used to produce it. However, one subset of breeding methods, generally referred to as recombinant DNA (rDNA) techniques, has been subject to intensive regulatory oversight and scrutiny since it was first introduced into plant and animal breeding programmes in the 1990s. In the case of genetically engineered animals, this regulatory burden has proven to be an insurmountable hurdle for all but a single application, the fast growing Atlantic salmon known as the AquAdvantage salmon which received formal approval from both US (17) and Canadian (18) regulatory authorities in 2015 and 2016, respectively. 
The original paper reporting the fast growing Atlantic salmon was published in 1992 (19); the final US approval was announced more than two decades later in December, 2015. The regulatory process associated with this product has been fraught with political complications and lengthy deliberations $(20,21)$. The expensive and prolonged regulatory journey of this first GE animal approved for food purposes has had a chilling effect on the use of genetic engineering by animal breeders worldwide. Animal breeding programmes make genetic improvement every generation, and a protracted regulatory delay in approving the products produced using specific breeding methods effectively impedes the use of such methods in breeding programmes.

Many international and national agencies are involved with the regulation and governance of genetically engineered organisms including the Food and Agriculture Organization of the United Nations (FAO)/World Health Organization (WHO), the World Organization for Animal Health (OIE), United States Food and Drug Administration (FDA), The Cartagena Protocol on Biosafety (CPB), the European Medicine Agency (EMA), and the European Food Safety Authority (EFSA). The definition of a "genetically engineered" animal differs among these different agencies.

The FAO and WHO established the Codex Alimentarius (Codex), or "Food Code" to develop international food standards that are harmonised and protect consumer health while also promoting fair practices in food trade. In 2008, the Codex developed internationally-recognised, science-based recommendations for evaluating the nutrition and safety of food from GE animals in the "Guideline for the Conduct of Food Safety Assessment of Foods Derived from Recombinant-DNA Animals (GL68-2008)" (22). These guidelines defined a "Recombinant-DNA Animal" as one in which the genetic material was changed through in vitro nucleic acid techniques, including rDNA and direct injection of nucleic acids into cells or organelles. The document clarifies that the terms GM and transgenic are used interchangeably to refer to animals that contain recombinant DNA, and that transgene refers to the recombinant DNA that has been integrated into the animal's genome. 
The World Organization for Animal Health (OIE) is an intergovernmental organization created to provide information regarding the global animal disease situation. Its standards are aimed at preventing the introduction of infectious agents and diseases through international trade. The standards of the OIE do not make specific reference to GE animals, but they would fall within their scope if the GE animal presented an animal health risk.

An international agreement, the Cartagena Protocol on Biosafety (CPB), aims to ensure the safe handling, transport and use of any living modified organism. "Living modified organism" is defined by the CPB as any living organism that possesses a novel combination of genetic material obtained through the use of modern biotechnology. It specifically excludes techniques used in traditional breeding and selection. "Modern biotechnology" means the application of: (1) in vitro nucleic acid techniques, including recombinant deoxyribonucleic acid (rDNA) and direct injection of nucleic acid into cells or organelles, or (2) fusion of cells beyond the taxonomic family that overcomes natural physiological reproductive or recombination barriers and that is not achieved through techniques used in traditional breeding and selection.

Similarly, EU Directive 2001/18/EC defines a genetically engineered organism as one, "with the exception of human beings, in which the genetic material has been altered in a way that does not occur naturally by mating and/or natural recombination". The Directive excludes techniques/methods of genetic modification if they do not involve the use of recombinant nucleic acid molecules or genetically modified organisms other than those produced by one or more of the techniques/methods of (1) mutagenesis, and (2) cell fusion (including protoplast fusion) of cells of organisms which can exchange genetic material through traditional breeding methods.

Lastly, the US Food and Drug Administration's definition of "genetically engineered (GE) animals" is those modified by rDNA techniques, including the entire lineage of animals that contain the modification (23). The rDNA construct in the GE animal is considered a new animal drug and thus is a regulated article under the new animal drug provisions of the Federal Food Drug and Cosmetics Act. Therefore it is not clear if it is the use of rDNA techniques in the development of 
a product, or the presence of an rDNA construct (drug) in the product, that is the trigger for regulatory oversight. These provisions apply to all GE animals regardless of their intended use.

Foreign rDNA or "transgenic" sequences are not necessarily introduced into the genome through the process of gene editing. In many cases the changes produced could not be distinguished from variations that occur naturally. As a result, the classical definitions of genetic engineering will not fit many gene editing applications. In some of the examples of applications for gene editing, the intent is to edit an allele using a nucleic acid template whose sequence is that of a naturally-occurring allele from the same species (e.g. hornless Holstein cattle edited to carry the polled allele from the Angus cattle breed) (13). In these cases, the genome of the edited animal will not contain a novel combination of genetic material, which would seem to exempt it from the CPB. There also would be no novel phenotype associated with the sequence. The unique risks that might be associated with an animal that is carrying such an allele are not evident given that the exact same sequence and resulting phenotype would be naturally-occurring within the same species (24).

The Codex and FDA definitions of 'Recombinant-DNA animals' are also awkward. They cover all animals produced using in vitro nucleic acid techniques, including rDNA and direct injection of nucleic acids into cells or organelles. This would appear to solely be a process-based definition; however, the scope clarifies that "transgenic animals" means recombinant-DNA animals and that transgene means the recombinant DNA that has been integrated in the genome of the recombinant-DNA animal. This leaves unanswered the question of whether an animal carrying no rDNA but produced using in vitro nucleic acid techniques is a recombinant-DNA animal.

Gene editing could conceptually introduce double stranded breaks at locations other than the intended locus, which could result in unintended alterations elsewhere in the genome (25). However, these off-target events are routine in mutagenesis breeding, which is not currently subject to regulatory oversight. These mutations would not be distinguishable from the spontaneous de novo mutations that are the basis of all genetic variation, although they can be minimised by 
careful selection of the guide sequence that targets the specific DNA sequence to be cut as well as the design of the gene editing reagents (26). Complete sequencing of hornless Holstein calves derived from two independent cell lines to 20X coverage did not find any off-target introgression of the polled allele, nor any insertion-deletions (indels) ascribable to off-target DNA cleavage by the nucleases and subsequent repair by non-homologous end joining within 10 base pairs of any of the identified degenerate targets (13).

Ultimately, the regulatory oversight of gene editing in food animals will establish the utility of this technique in structured livestock breeding programmes. One feature of many animal industries is the exploitation of heterosis, or hybrid vigor, in commercial animals through the development of a four-way cross from distinct elite parent lines. This means that if an edited allele is to be introduced, it is likely that multiple parent lines will need to be separately modified using genome editing to maintain the genetic diversity in the founder lines that results in the heterotic advantage of the hybrid. This is especially true for traits with a recessive mode of inheritance as animals will need to be homozygous for the desired recessive allele. Introgression of these genetic variations via repeated backcrossing from non-parent germplasm is not feasible given the long generation interval of most livestock species. Each generation of an animal breeding programme is genetically superior to the former, and gene editing will need to seamlessly integrate into such programmes if it is to be employed. As such, the lengthy eventbased approval paradigm that has been the hallmark of plant genetic engineering regulations, meaning each insertion event of a specific rDNA construct in a given species and specific genomic location is a separate multiyear regulatory evaluation, would likely preclude the use of gene editing in animal genetic improvement programmes.

Recently, a number of policy papers discussing the need for regulation of genome editing to be science-based, proportional to risk, product focused and fit for purpose have been published by normally inconspicuous public sector breeders and academics from around the world (24, 2731). The US National Academy of Sciences recently (32) concluded that a clear distinction between conventional breeding and genetic engineering is becoming less obvious and that the 
same benefits and risks are associated with conventionally bred and genetically engineered varieties. They further concluded that as the old approaches to genetic engineering become less novel and as emerging processes (such as genome editing and synthetic biology) fail to fit current regulatory categories of genetic engineering, a process-based regulatory approach is becoming less and less technically defensible. As a result, they recommended a tiered regulatory approach that focuses on any intended and unintended novel characteristics of the end product that result from the breeding methods that may present potential hazards instead of focusing regulation on the process or breeding method used to achieve the genetic change.

\section{WHY DOES THIS MATTER?}

While this question of regulation of genome editing in animals might seem academic, the ultimate outcome of these deliberations is of global importance. Conventional plant and animal breeders have been unobtrusively contributing to the sustainability of food production for the past century. Genetics may not be immediately associated with the term sustainability, yet the importance of animal genetics in contributing to the interplay between the environmental, social and economic goals of sustainability should not be underrated. Genetic gains are both permanent and cumulative meaning that gains made in one year will be transmitted to subsequent generations without further endeavour or expenditure.

Perhaps no other breeding method has had as great an impact on accelerating the rate of genetic gain through increasing the intensity of selection as artificial insemination (Al). Al technology was introduced into the dairy industry and commercialised in the United States during the late 1930s to early 1940s. Seventy percent of all dairy cows in the US are bred using Al, as are virtually all turkeys and chickens. Artificial insemination allows the extensive use of high-accuracy, genetically superior sires and plays a major role in design of breeding programmes and dissemination of advanced genetics.

Although Al is now used routinely in animal breeding and human medicine, it was initially viewed with scepticism. There was a fear that Al would lead to abnormalities, and influential cattle 
breeders were originally opposed to the concept as they believed it would destroy their bull market (33). When independent, university research demonstrated that the technology could be used to provide superior bulls, control venereal disease, and produce healthy calves, adoption was swift.

To put the impact of the genetic improvement enabled by $\mathrm{Al}$ in a sustainability perspective, consider that advances in the genetics, nutrition and management of US dairy cows over the last century have resulted in a greater than four-fold increase in milk production per cow, and a threefold improvement in production efficiency (milk output per feed resource input (34)). About half of this $369 \%$ increase in production efficiency is attributable to genetic improvement enabled by Al. As a result, today a much smaller population of dairy cows supplies the US market. The US dairy cattle population peaked in 1944 at an estimated 25.6 million animals with a total annual milk production of approximately 53.1 billion $\mathrm{kg}$. In 1997, dairy cattle numbers had decreased to 9.2 million animals and total annual production had increased to an estimated 70.8 billion $\mathrm{kg}$. The availability of frozen semen also dramatically curtailed the number of natural service dairy bulls on farms which further lessened the inputs required to produce a unit of milk (35).

From an environmental perspective, genetic improvement over the past 50 years has resulted in dramatic reductions in greenhouse gas (GHG) emissions and global warming potential per ton of animal product. Capper et al. (35) reported that although the carbon footprint per individual cow increased when comparing 1944 to 2007, increases in milk production per cow mean that the carbon footprint per unit of milk in 2007 was one third what it was in 1944.

Precluding the adoption of innovations in animal breeding programmes is associated with considerable opportunity costs. These tradeoffs must be considered in decisions about the risks and (potential) benefits of new technologies. Both the current US regulatory approach and the precautionary principle do not explicitly consider potential benefits of the technology which may include improvements to animal welfare (e.g. disease-resistant animals), enhanced sustainability and human health. Consideration of such benefits would represent a shift away from a risk- 
assessment process that focuses on identifying ever diminishing marginal hazards, to one that addresses the probability of whether the benefits outweigh the risks (36). At the current time there are no international standards for assessing the benefits associated with GMOs, although in many countries there are increasing calls for a risk-benefit analysis to be included in regulatory frameworks (37).

The "risk-only" analysis that is entrenched in the current GE animal regulatory review is problematic as no activity is free of risk. Evenly applied to biomedical applications of GE animals, a "risk-only" analysis would likely have prevented the commercialization of the recombinant therapeutic proteins ATryn $^{\circledR}$ (human antithrombin-III) and the Ruconest ${ }^{\mathrm{TM}}$ (Rhucin ${ }^{\circledR}$ outside the EU) produced in GE goats and rabbits, respectively, as they may be associated with some risks. In these cases the benefits to the human patients needing the medications were judged to outweigh any potential risks that might be associated with production in GE animals. An analogous evaluation should be associated with genetic modifications of animals for food applications.

Ideally, the regulation of gene edited animals would be considered on a case-by-case basis depending upon the novelty associated with the phenotypes displayed by, or traits imbued to, the edited animal. The extent of regulatory oversight needs to be proportional to the unique risks, if any, associated with the novel phenotypes weighed against potential benefits. Animal breeders are perhaps the group that are most aware of the stifling impact of regulatory gridlock on the deployment of potentially valuable breeding techniques given there is not yet a single genetically engineered animal being sold for food anywhere in the world.

Ideally, the best regulatory approach is one that allows new technologies to be used while preventing unacceptable risks to animal and human health or the environment. Here the definition of unacceptable becomes contentious, with some arguing that any level of risk is unacceptable. However, in a world facing burgeoning animal protein demands, it is important to ensure that regulatory frameworks also appropriately consider and weigh the potential benefits of gene edited animals to global food security. Perhaps, as importantly should be a careful 
evaluation of the proposed regulatory approach to ensure that it is fit for purpose, meaning that the reduction in risk obtained by regulatory oversight is greater than the costs of compliance.

\section{COMPETING INTERESTS}

The author declares no competing interests.

\section{FUNDING}

The author's gene editing research is supported by the Biotechnology Risk Assessment Grant Program Competitive Grant no. 2015-33522-24106 and Agriculture and Food Research Initiative Competitive Grant no. 2015-67015-23316 from the National Institute of Food and Agriculture/U.S. Department of Agriculture.

\section{REFERENCES}

1. Cotter J, Zimmermann D, van Bekkem H. Application of the EU and Cartagena definitions of a GMO to the classification of plants developed by cisgenesis and gene-editing techniques. Greenpeace Research Laboratories Technical Report (Review); 2015.

2. Kasinathan $P$, Wei $H$, Xiang T, Molina JA, Metzger J, Broek D, et al. Acceleration of genetic gain in cattle by reduction of generation interval. Scientific Reports. 2015;5:8674.

3. Reyes LM, Estrada JL, Wang ZY, Blosser RJ, Smith RF, Sidner RA, et al. Creating class I MHC-null pigs using guide RNA and the Cas9 endonuclease. Journal of immunology (Baltimore, Md : 1950). 2014;193(11):5751-7.

4. Li P, Estrada JL, Burlak C, Montgomery J, Butler JR, Santos RM, et al. Efficient generation of genetically distinct pigs in a single pregnancy using multiplexed single-guide RNA and carbohydrate selection. Xenotransplantation. 2015;22(1):20-31.

5. Tan W, Proudfoot C, Lillico SG, Whitelaw CB. Gene targeting, genome editing: from Dolly to editors. Transgenic research. 2016; 25(3):273-87.

6. Keefer CL. Artificial cloning of domestic animals. Proceedings of the National Academy of Sciences. 2015;112(29):8874-8. 
7. Peng J, Wang $Y$, Jiang J, Zhou X, Song L, Wang L, et al. Production of Human Albumin in Pigs Through CRISPR/Cas9-Mediated Knockin of Human cDNA into Swine Albumin Locus in the Zygotes. Scientific Reports. 2015;5:16705.

8. Lillico SG, Proudfoot C, King TJ, Tan W, Zhang L, Mardjuki R, et al. Mammalian interspecies substitution of immune modulatory alleles by genome editing. Scientific reports. 2016;6:21645.

9. Han H, Ma Y, Wang T, Lian L, Tian X, Hu R, et al. One-step generation of myostatin gene knockout sheep via the CRISPR/Cas9 system. Frontiers of Agricultural Science and Engineering. 2014;1(1):2-5.

10. Lillico SG, Proudfoot C, Carlson DF, Stverakova D, Neil C, Blain C, et al. Live pigs produced from genome edited zygotes. Sci Rep. 2013 ; 3:2847.

11. Proudfoot C, Carlson DF, Huddart R, Long CR, Pryor JH, King TJ. Genome edited sheep and cattle. Transgenic research. 2015; 24(1):147-53.

12. Van Raden P, Olson K, Null DJ, Hutchison J. Harmful recessive effects on fertility detected by ansence of homozygous haplotypes. Journal of Dairy Science. 2011;94:6153-61.

13. Carlson DF, Lancto CA, Zang B, Kim E-S, Walton M, Oldeschulte D, et al. Production of hornless dairy cattle from genome-edited cell lines. Nat Biotech. 2016;34(5):479-81.

14. Gholap P, Kale D, Sirothia A. Genetic diseases in cattle: A review. Research Journal of Animal, Veterinary and Fishery Sciences. 2014;2:24-33.

15. Hickey JM. Sequencing millions of animals for genomic selection 2.0. J Anim Breed Genet. 2013;130.

16. Jenko J, Gorjanc G, Cleveland MA, Varshney RK, Whitelaw CBA, Woolliams JA, et al. Potential of promotion of alleles by genome editing to improve quantitative traits in livestock breeding programs. Genetics Selection Evolution. 2015;47(1):1-14.

17. Waltz E. GM salmon declared fit for dinner plates. Nat Biotech. 2016;34(1):7-9.

18. Health Canada, Health Canada and Canadian Food Inspection Agency approve AquAdvantage salmon. 2016. Ottawa, ON (CAN): Government of Canada; 2016. Available from: http://news.gc.ca/web/article-en.do?nid=1068309\&tp=980. Accessed November 2, 2016. 
19. Du SJ, Gong Z, Fletcher GL, Shears MA, King MJ, Idler DR, et al. Growth enhancement in transgenic Atlantic salmon by the use of an "all fish" chimeric growth hormone gene construct. Nat Biotechnol. 1992;10(2):176-81.

20. Fox JL. Transgenic salmon inches toward finish line. Nat Biotech. 2010;28(11):1141-2.

21. Van Eenennaam AL, Muir WM. Transgenic salmon: a final leap to the grocery shelf? Nat Biotechnol. 2011;29(8):706-10.

22. Guideline for the conduct of food safety assessment of foods derived from recombinant-DNA animals, http://www.codexalimentarius.net/download/standards/11023/CXG_068e.pdf (2008). 23. Food and Drug Administration (FDA). Regulation of Genetically Engineered Animals Containing Heritable Recombinant DNA Constructs. http://www.fda.gov/downloads/AnimalVeterinary/GuidanceComplianceEnforcement/Guidance forlndustry/UCM113903.pdf U.S. Department of Health and Human Services; 2009.

24. Carroll D, Van Eenennaam AL, Taylor JF, Seger J, Voytas DF. Regulate genome-edited products, not genome editing itself. Nat Biotech. 2016;34(5):477-9.

25. Koo T, Lee J, Kim JS. Measuring and Reducing Off-Target Activities of Programmable Nucleases Including CRISPR-Cas9. Molecules and cells. 2015;38(6):475-81.

26. O'Geen H, Yu AS, Segal DJ. How specific is CRISPR/Cas9 really? Current Opinion in Chemical Biology. 2015;29:72-8.

27. Bruce A, Castle D, Gibbs C, Tait J, Whitelaw CB. Novel GM animal technologies and their governance. Transgenic research. 2013;22(4):681-95.

28. Camacho A, Van Deynze A, Chi-Ham C, Bennett AB. Genetically engineered crops that fly under the US regulatory radar. Nat Biotech. 2014:1087-91.

29. Conko G, Kershen DL, Miller H, Parrott WA. A risk-based approach to the regulation of genetically engineered organisms. Nat Biotech. 2016;34(5):493-503.

30. Murray JD, Maga EA. Opinion: A new paradigm for regulating genetically engineered animals that are used as food. Proceedings of the National Academy of Sciences. 2016;113(13):3410-3. 31. Van Eenennaam AL, Young AE. Animal agriculture and the importance of agnostic governance of biotechnology. Agriculture \& Food Security. 2015;4(1):1-10. 
32. NAS (National Academies of Sciences Engineering and Medicine). Genetically Engineered Crops: Experiences and Prospects. Washington, DC: The National Academies Press., 2016.

33. Foote RH. The history of artificial insemination: Selected notes and notables. Journal of Animal Science. 2002 January 1, 2002;80(E-Suppl 2):1-10.

34. VandeHaar MJ, St-Pierre N. Major Advances in Nutrition: Relevance to the Sustainability of the Dairy Industry. Journal of Dairy Science. 2006;89(4):1280-91.

35. Capper JL, Cady RA, Bauman DE. The environmental impact of dairy production: 1944 compared with 2007. Journal of Animal Science. 2009;87(6):2160-7.

36. Van Eenennaam AL. GMOs in animal agriculture: time to consider both costs and benefits in regulatory evaluations. Journal of Animal Science and Biotechnology. 2013;4(1):37.

37. Morris EJ. A semi-quantitative approach to GMO risk-benefit analysis. Transgenic research. 2011;20(5):1055-71. 